\title{
Transcriptome assembly and expression profiling of the molecular responses to cadmium toxicity in cerebral ganglia of wolf spider Pardosa pseudoannulata (Araneae: Lycosidae)
}

\author{
Huilin Yang ${ }^{1,2} \cdot$ Yuande Peng ${ }^{3} \cdot$ Yixue Shi $^{2} \cdot$ Jianxiang Tian $^{4} \cdot \mathrm{Juan} \mathrm{Wang}^{2}$ •

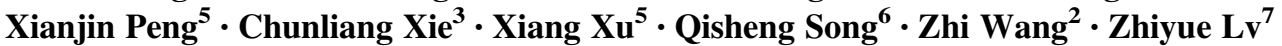

Accepted: 5 December 2017 / Published online: 3 January 2018

(C) The Author(s) 2018. This article is an open access publication

\begin{abstract}
Cadmium (Cd) is a heavy metal that can cause irreversible toxicity to animals, and is an environmental pollutant in farmlands. Spiders are considered to be an excellent model for investigating the impacts of heavy metals on the environment. To date, the changes at the molecular level in the cerebral ganglia of spiders are poorly understood. Cd exposure leads to strong damage in the nervous system, such as apoptosis and necrosis of nerve cells, therefore we conducted a transcriptomic analysis of Pardosa pseudoannulata cerebral ganglia under $\mathrm{Cd}$ stress to profile differential gene expression (DGE). We obtained a
\end{abstract}

Electronic supplementary material The online version of this article (https://doi.org/10.1007/s10646-017-1885-1) contains supplementary material, which is available to authorized users.

\section{Zhi Wang \\ wangzhispider@hotmail.com \\ $₫$ Zhiyue Lv \\ lvzhiyue@mail.sysu.edu.cn}

1 College of Orient Science \& Technology, Hunan Agriculture University, No. 1 Nongda Road, Changsha 410128 Hunan, China

2 College of Bioscience and Biotechnology, Hunan Agriculture University, No. 1 Nongda Road, Changsha 410128 Hunan, China

3 Institute of Bast Fiber Crops, Chinese Academy of Agricultural Sciences, Changsha 410205 Hunan China

4 College of Continuing Education, Hunan Agriculture University, No. 1 Nongda Road, Changsha 410128 Hunan, China

5 College of Life Science, Hunan Normal University, Changsha 410006 Hunan, China

6 Division of Plant Sciences, University of Missouri, Columbia, MO 65211, USA

7 Department of Parasitology, Zhongshan School of Medicine, Sun Yat-Sen University, Guangzhou 510080, China total of 123,328 assembled unigenes, and $1441 \mathrm{Cd}$ stressassociated DEGs between the Cd-treated and control groups. Expression profile analysis demonstrated that many genes involved in calcium signaling, cGMP-PKG signaling, tyrosine metabolism, phototransduction-fly, melanogenesis and isoquinoline alkaloid biosynthesis were upregulated under $\mathrm{Cd}$ stress, whereas oxidative phosphorylation-related, nervous disease-associated, non-alcoholic fatty liver disease-associated, and ribosomal-associated genes were down-regulated. Here, we provide a comprehensive set of DEGs influenced by Cd stress, and heavy metal stress, and provide new information for elucidating the neurotoxic mechanisms of $\mathrm{Cd}$ stress in spiders.

Keywords Cadmium $\cdot$ Transcriptomic $\cdot P$. pseudoannulata $\cdot$ Cerebral ganglion

\section{Introduction}

Cadmium (Cd) is a non-essential heavy metal that is a nonbiodegradable pollutant across the world (Mehinto et al. 2014; Nemmiche et al. 2011). Cd is highly neurotoxic in animals, and damages induced by high levels of $\mathrm{Cd}$ include changes in brain morphology, disruption of the brain barrier of the central nervous system, changes in neurotransmitter content and enzyme activity, and alterations in brain metabolism (Murthy et al. 1989). Interestingly, Cd toxicity can have different effects within individuals of the same species. For example, Cd distribution in the ganglia is different in various strains of mice, resulting in differing effects of $\mathrm{Cd}$ to the trigeminal ganglia (Habeebu et al. 2001).

Spiders are considered to be an excellent invertebrate model to investigate $\mathrm{Cd}$ contamination, based on species diversity, geographic distribution and large appetite (Jung et al. 2005; 
Yang et al. 2016; Li et al. 2016). Previous studies have demonstrated that $\mathrm{Cd}$ can accumulate in spiders and affect their physiological and ecological traits (Wilczek et al. 2008; Jung and Lee 2012; Jöst and Zauke 2008; Eraly et al. 2010). More than 5000 farmland spider species belonging to 3000 genera have been described in the world, and these spiders are often the most generalist predator in farmlands (Samu and Szinetár 2002). Pardosa pseudoannulata belongs to Lycosidae, and is one of the most common wandering spiders in fields across the rice growing regions of China ( $\mathrm{Li}$ et al. 2001). Li et al. (2016) carried out a whole body transcriptome analysis of the spider $P$. pseudoannulata exposed to $\mathrm{Cd}$, and identified a total of 2939 differentially expressed genes (DEGs), including multiple candidate genes involved in defense and detoxification of $\mathrm{Cd}$, as well as genes encoding glutathione metabolism related enzymes and heat shock proteins.

The rapid development of high-throughput DNA sequencing technology has facilitated detailed and comprehensive analysis of the transcriptomes and genomes of non-model organisms (Margulies et al. 2005; Wicker et al. 2006; Liu et al. 2013; Wang et al. 2016a, 2017). Meng et al. (2013) found over 3000 differentially expressed genes (DEGs) in the digestive glands of Japanese scallops Mizuhopecten yessoensis upon $\mathrm{Cd}$ exposure, of which 154 DEGs were involved in $\mathrm{ABC}$ transporters, glycine, serine and threonine metabolism, steroid hormone biosynthesis and glutathione metabolism. Sun et al. (2016) conducted transcriptomic analysis of Cd-treated freshwater crab hepatopancreas tissues, and reported increases in expression of genes involved in macromolecular metabolism, oxidative phosphorylation, detoxification and anti-oxidant defense. In the hepatopancreas of the razor clam Sinonovacula constricta exposed to Cd, genes showing significant expression level changes included those involved in metabolic processes, cellular processes, and ROS production-related genes, such as heat shock proteins 32, metallothionein, and glutathione (Wang et al. 2016b).

Here, we conducted a comparative analysis of DGEs in the nervous system of $P$. pseudoannulata in response to $\mathrm{Cd}$ stress to identify associated biological processes and pathways, and to reveal the type of processes that may be disrupted in the cerebral ganglion of spider. To our knowledge, this is the first report on the transcriptome profiling of the cerebral ganglion of $P$. pseudoannulata, thus improving our understanding of the mechanism by which heavy metal neurotoxins impact the spider.

\section{Materials and methods}

\section{Animal materials and treatments}

P. pseudoannulata specimens were collected from experimental farmland in the Hunan Academy of Agricultural
Science, Changsha $\left(27^{\circ} 51^{\prime} \mathrm{N}, 111^{\circ} 53^{\prime} \mathrm{E}\right)$, Hunan Province, China. Spiders were placed individually in cylindrical glass tubes with a moist cotton ball $(12 \times 100 \mathrm{~mm})$. Spiders were fed daily with Drosophila melanogaster that were reared on cadmium chloride medium $(1.0 \mathrm{mg} / \mathrm{L})$ or non-Cd medium. All tubes were marked and maintained in an artificial climate chamber maintained at $30{ }^{\circ} \mathrm{C}, 70 \% \mathrm{RH}$ and L:D 10:14 photoperiod.

A total of 120 spiders were used in the experiment. To determine $\mathrm{Cd}$ concentrations, there was a control group and an experimental group each with three replicates with each replicate containing 20 spiders. Spiders were observed twice a day at 9 a.m. and 9 p.m. No mortality was observed during the entire experimental period. The spiders were dissected within an ice bath after 30 days of treatment, the cerebral ganglion in the Cd-treated and control spiders were collected, immediately frozen in liquid nitrogen and stored at $-70{ }^{\circ} \mathrm{C}$ for RNA extraction.

\section{Spider sample digestion and $\mathrm{Cd}$ content determination}

Each sample weighed between $0.2-0.5 \mathrm{~g}$ and the samples were washed with $1 \%$ nitric acid three times, then placed into a tube containing $1.5 \mathrm{~mL}$ hydrochloric acid and $4.5 \mathrm{~mL}$ nitric acid for digestion. The samples were digested by a digestion instrument (SPH620, Alva instrument) at $90^{\circ} \mathrm{C}$ for $1 \mathrm{~h}$ and $120^{\circ} \mathrm{C}$ for $2 \mathrm{~h}$, and then the cadmium content of the samples were determined by ICP (ICPE-9000) at $228.3 \mathrm{~nm}$.

\section{RNA extraction, transcriptome sequencing and de novo assembly}

RNA extraction and transcriptome sequencing were conducted by Oebiotech Enterprise, Shanghai. RNA was extracted from a pooled samples of 40 cerebral ganglions dissected from the experimental or control spiders. The quality of RNAs were determined with a NanoDrop ND1000 spectrophotometer (NanoDrop Technologies Inc., Rockland, DE, USA), and only samples with $1.8 \leq$ OD260/ OD280 $\leq 2.1$ were used for generating the transcriptome. RNA sequencing libraries were constructed and sequenced on an Illumina Hiseq ${ }^{\mathrm{TM}} 2500$.

Raw sequences were obtained by removing adapter sequences and low quality sequences by TGICL (Pertea et al. 2003). The remaining clean reads were used for de novo assembly by Trinity (Grabherr et al. 2011). Unique unigenes were generated by removing redundant sequences, and these unigenes were used for downstream bioinformatics analysis.

\section{Functional annotation}

All unigenes were compared to protein databases, including the NCBI non-redundant protein $(\mathrm{Nr})$ database and Swiss- 
prot database using Blastx. The Blast2GO program and WEGO software were used to obtain GO (Gene ontology) annotation for all unigenes (Conesa et al. 2005; Ye et al. 2006). COG (clusters of orthologous group) classification and KEGG (Kyoto encyclopedia of genes and genomes database) metabolic pathway annotation of unigenes were assigned by Blastx searching against KEGG and COG databases (Tatusov et al. 2003). The best aligned results were used to determine potential function of the unigenes. The parameter E-value $<1 \mathrm{e}-5$ of BLASTx was taken as a threshold of significant similarity.

\section{Identification of differentially expressed genes (DEGs) and functional annotation}

The FPKM (fragments per kb per million reads) method was used to quantify gene expression levels (Trapnell et al. 2010). The DEGseq software package was used to screen DEGs using the negative binomial distribution algorithm (Wang et al. 2010). False discovery rate (FDR) $<0.01$ was used as the threshold to determine significant differences in gene expression. GO and KEGG pathway analyses were conducted for all DEGs. The hypergeometric test was used to identify significantly enriched GO terms in DEGs. The calculated $p$ values were then corrected using the Bonferroni Correction, with corrected $p$ value $\leq 0.001$ as a threshold. GO terms fulfilling this condition were defined as significantly enriched GO terms in DEGs.

Similarly, pathway enrichment analysis was conducted to identify significantly enriched pathways including metabolic pathways or signal transduction pathways in DEGs, using a $p$ value of $\leq 0.05$ as a threshold.

\section{Quantitative real-time PCR analysis}

Quantitative real-time PCR (qPCR) was used to verify the transcriptome results. Total RNA was extracted from each sample with TRIzol (Invitrogen, USA) and subjected to
DNase I treatment (Promega, USA) according to the manufacturers' protocols. cDNA was synthesized with a RevertAid $^{\text {TM }}$ H Minus First Strand cDNA Synthesis Kit (Fermentas Lithuania) and qPCR was performed using the ABI 7900 HT system (ABI, USA). The experiment was repeated three times, and expression levels of each gene were normalized to $18 \mathrm{~S}$ ribosomal RNA and calculated using the $2^{-\Delta \Delta \mathrm{Ct}}$ method. All primers were designed using the using the Primer 3.0 program (Table 1).

qPCR data were analyzed using a $t$-test with SPSS 17.0 software. Significant differences at $p<0.05$ were designated with *, and data were presented as the mean \pm SE.

\section{Results}

\section{Amount of cadmium (Cd) in P. pseudoannulata}

To test the effect of $\mathrm{Cd}$ on $P$. pseudoannulata, we fed spiders with fruit flies that were reared on standard media (control), or Cd-containing media (treatment) continuously for 30 days. Control spiders did not contain any detectable $\mathrm{Cd}$. Cd treated spiders had increasing $\mathrm{Cd}$ levels with feeding time, reaching $7.27 \mu \mathrm{g} / \mathrm{g}$ Cd by day 30 .

\section{De novo assembly of unigenes}

We generated transcriptomes of $P$. pseudoannulata cerebral ganglion of treated and control spiders and obtained $47,661,402$ clean DNA sequences reads with 5,957,675,250 bases after removing the low-quality reads, with Q30 percentages of $92.10 \%$. De novo assembly of the clean reads was performed and 123,328 unigenes with an average length of $1040.73 \mathrm{bp}$ were obtained. The $\mathrm{N}^{5} 0_{\mathrm{s}}$ of the unigenes were 1554, and 36,991 unigenes were over $1 \mathrm{~kb}$ (Table 2).

Table 1 List of primers used for qPCR

\begin{tabular}{llll}
\hline No. & Gene symbol & Forward primer & Reverse primer \\
\hline 1 & CL9578Contig1 & GAATTTCGACGAGATAGACCG & TTATTCTTTCCTGCCACGC \\
2 & CL5353Contig1 & CACACTGTCTACAAAGTCCTG & GAACCACGAATTGGGAGAT \\
3 & CL8937Contig1 & CCAACGCAGACAATCAGAAGA & ACGACAGGATCAGGTAGG \\
4 & CL911Contig1 & GTCTGCCTGTCTTTCCTTG & TTCAGCAGTAACACTCTCGTAG \\
5 & CL14434Contig1 & CAGCTTCGACGAGTTCAG & ATGTAAGTCTCGATCACGTTG \\
6 & comp228568_c0_seq3 & CTGCTGCTATGTAAGGATGTCA & ACTCTTGGCTGCTTTGGAA \\
7 & CL17095Contig1 & GCTACAACGATCTCTTAGCCT & TAGCCGTCCAGATAGTTTGC \\
8 & CL1Contig1327 & GCTCCTTCGCTGTTTAGTC & GCAGAGAAAGTTGTTGGCA \\
9 & comp42711_c0_seq2 & AGTGCATTCAAGTGAAGGT & ATCATCAACACATTTGAACAGC \\
10 & CL21551Contig1 & CCGAACAGGCTCAAGAAG & TCACGGAACCTCCGTAGATA \\
11 & 18 S & CGGCTACCACATCCAAGGAA & GCTGGAATTACCGCGGCT \\
\hline
\end{tabular}




\section{Functional annotation}

Among all unigenes, 39,029 (31.65\% of all unigenes) matched to the Nr database, $28,049(22.74 \%)$ to the Swiss-prot database, $25,031(20.30 \%)$ to COG, $25,996(21.08 \%)$ to the GO database, and $10,254(8.31 \%)$ to the KEGG database (Table 3 ). From the results of annotation of the $\mathrm{Nr}$ database, 454 unigenes $(1.16 \%$ of the total) showed similar sequences with the genome of Zootermopsis nevadensis, 442 unigenes (1.13\% of the total) with the genome of Acyrthosiphon pisum, 358 unigenes $(0.92 \%$ of the total) with the genome of Ixodes scapularis. A total of 28,113 unigenes $(72.04 \%)$ did not match those in any species. GO annotation enriched unigenes were divided into three clusters: biological process (BP), cellular component (CC) and molecular function (MF). Biological processes made up the majority of the annotated unigenes (66.71\%), followed by molecular functions $(22.79 \%)$ and cell components $(10.49 \%)$. The major subcategories were "cell" and "cell part" in cell components, and "cellular process" within biological processes (Fig. 1).

A total of 25,031 unigenes had significant matches in the COG database. Among these, the "General function prediction only" cluster $(21.37 \%)$ was the largest, followed by "Signal transduction mechanisms" (18.04\%), "Post-translation modification, protein turnover, chaperones", and "transcription" (7.83\%) (Fig. 2). In addition, 10,254 unigenes were categorized into 333 KEGG pathways. Five largest categories were pathways in cancer (human diseases/cancers), ribosome (genetic information processing/translation), Huntington's disease (human diseases/ neurodegenerative diseases), protein processing in endoplasmic reticulum (genetic information processing/folding, sorting and degradation), and lysosome (cellular processes/ transport and catabolism).

\section{Differential gene expression}

A total of 1441 DEGs were identified, of which 174 were significantly up-regulated and 1267 were significantly down-regulated $(p \leq 0.01)$. Among all DEGs, 126 had fold changes ranging from -5 to -10 and the remaining 46 had fold changes ranging from 5 to 10 (Fig. 3). These results show that $\mathrm{Cd}$ treatment altered gene expression in the cerebral ganglion of Cd-treated spiders.

\section{Functional enrichment analysis of DEGs}

To explore the expression of DEGs in the ganglia of spiders in the present study, we conducted GO term functional annotation based on GO classification and pathway enrichment analysis. We found that $48 \mathrm{GO}$ terms (FDR $<$ $0.001)$ were significantly enriched. Among the GO term functional annotation, biological process (BP) (26 GO terms, 102 genes), cellular component (CC) (8 GO terms, 304 genes) and molecular function (MF) (14 GO terms, 141 genes) accounted for $54.16,16.67$ and $29.17 \%$ respectively (Figs. 1-3). The main enriched subcategories in biological process were "proteolysis involved in cellular protein catabolic process", "cellular iron ion homeostasis" and "phototransduction". The two main categories within the molecular function category were "structural constituent of cuticle" and "chitin binding", accounting for $41.14 \%$ of the DEGs in this category. In the cellular component category, "extracellular region" and "endoplasmic reticulum membrane" were the main enriched subcategories $(51.46 \%)$.

Top GOs were further used to identify enriched GO terms (Fig. 4). The predominantly enriched subcategories were "ion transport" (GO:0006811, part of BP), "extracellular region" (GO:0005576, part of CC), and "structural constituent of cuticle" (GO:0042302, part of MF). Among the up-regulated genes, the predominant enriched subcategory was "extracellular region", which accounted for $34.42 \%$ of the total number of up-regulated genes. For down-regulated genes, "extracellular exosome" (11.07\%) (FDR $<0.001)$ was predominantly enriched. These results suggest that $\mathrm{Cd}$ may induce damage to cellular components and their related functions in nervous tissue of spiders.

The KEGG annotation of DEGs identified 271 differently pathways, where 39 significantly enriched pathways were obtained. Under Cd stress, signaling transduction,
Table 2 Summary statistics of de novo transcriptome assembly

Table 3 Functional annotation of cerebral ganglion of $P$. pseudoannulata transcriptome

\begin{tabular}{lllllllll}
\hline $\begin{array}{l}\text { All } \geq 200 \\
\text { bp }\end{array}$ & $\geq 500$ bp & $\geq 1000$ bp & N50 & Total length & $\begin{array}{l}\text { Max } \\
\text { length }\end{array}$ & $\begin{array}{l}\text { Min } \\
\text { length }\end{array}$ & $\begin{array}{l}\text { Average } \\
\text { length }\end{array}$ \\
\hline Unigene & 123,328 & 76,190 & 36,991 & 1554 & $128,350,740$ & 23,679 & 301 & 1040.73 \\
\hline
\end{tabular}

\begin{tabular}{llllll}
\hline Database & NR & SWISSPROT & KOG & KEGG & GO \\
\hline Annotation_numbers & 39,029 & 28,049 & 25,031 & 10,254 & 25,996 \\
Annotation_ratio & $31.65 \%$ & $22.74 \%$ & $20.30 \%$ & $8.31 \%$ & $21.08 \%$ \\
\hline
\end{tabular}




\section{gene ontology classification}

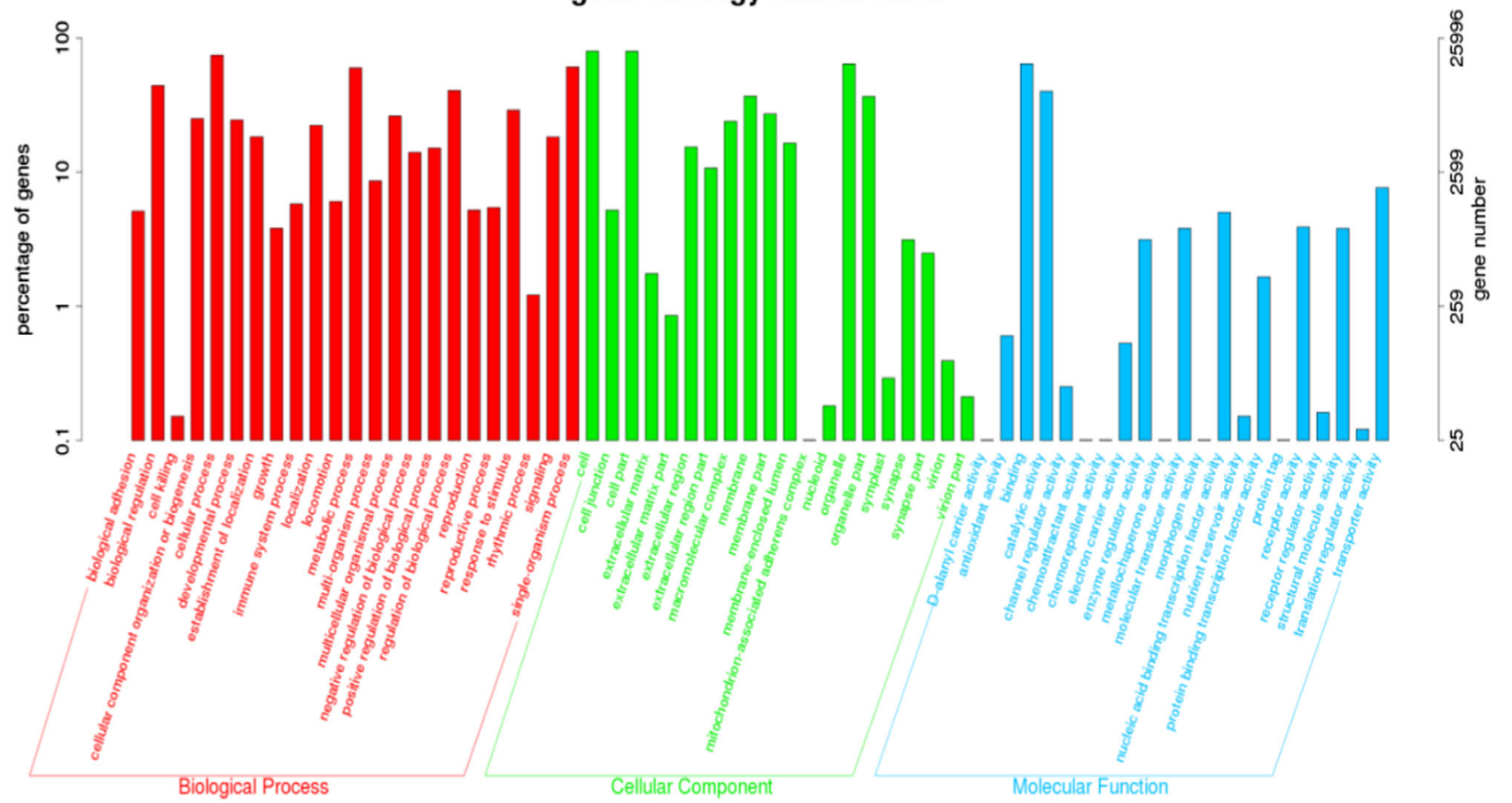

Fig. 1 GO classification of all unigenes identified in the transcriptome of the cerebral ganglia of $P$. pseudoannulata. Analysis was conducted using Blast2Go (level 2), and red bars represent GO processes under biological processes, green under cellular component, and blue under molecular function

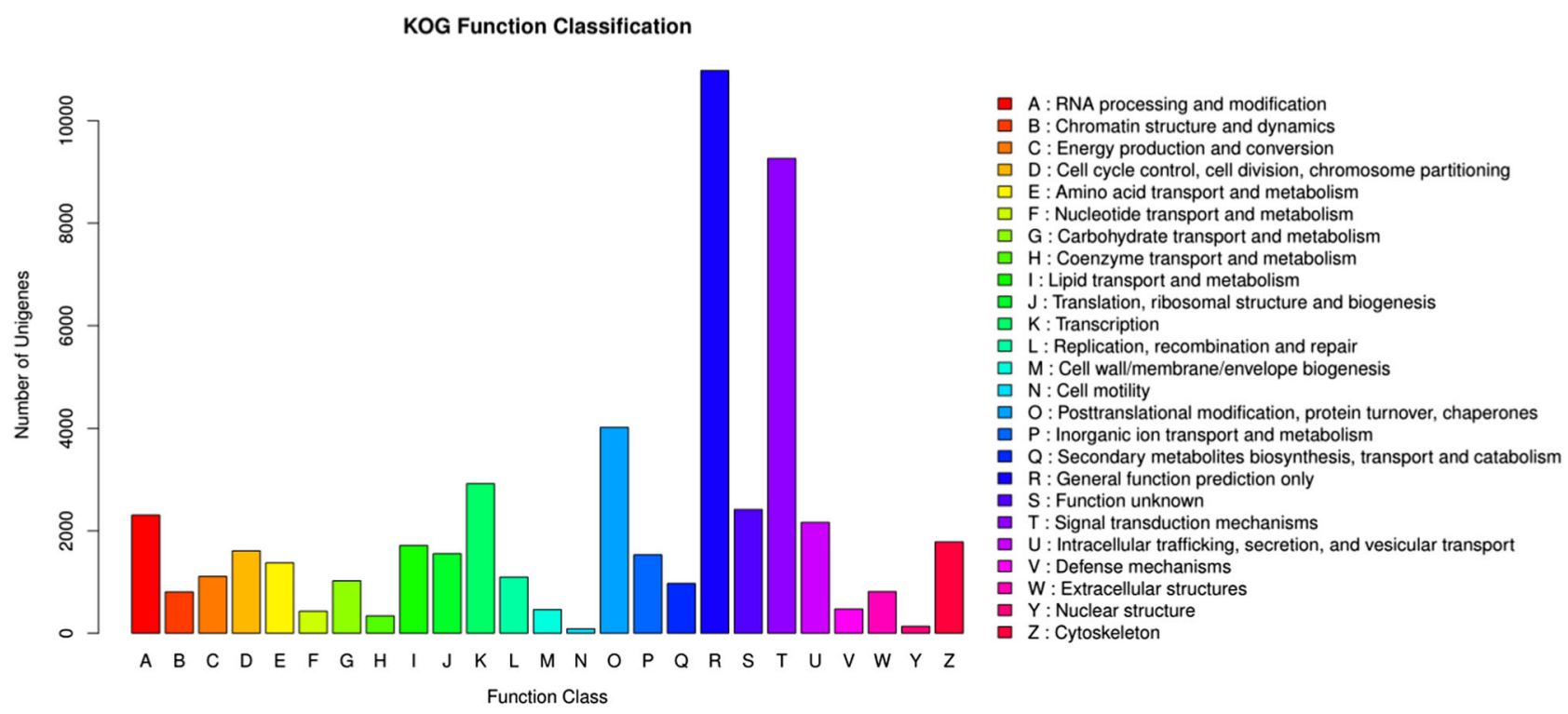

Fig. 2 COG classification of unigenes in the cerebral ganglion transcriptome of $P$. pseudoannulata

energy supply processing, protein processing, and nervous diseases were significantly enriched (Fig. 5, Table 4). The results showed that the pathway with the lowest $\mathrm{Q}$ value was Parkinson's disease, followed by Oxidative phosphorylation. Up-regulated pathways with maximum gene number were Calcium signaling pathway and cGMP-PKG signaling pathway, while down-regulated pathways were
Oxidative phosphorylation and Parkinson's disease (Table 4).

\section{qPCR validation of transcriptome analysis}

In order to verify the accuracy of the differential expression analysis of the transcriptome, four up-regulated or down- 
regulated genes were randomly selected for qPCR analysis. The qPCR results revealed that in the test group the expression of Comp208328_c0_seq4Contig1 transcript was 10.7 times higher than that in the control group, while the down-regulated genes Comp91669_c0_seq1, Comp42711_c0_seq2 and Comp215590_c0_seq2 Contig1 transcripts were only $0.16,0.11$ and 0.2 fold of that in the control group, respectively (Fig. 6). The results are consistent with those observed in the transcriptome analysis, demonstrating the repeatability of our transcriptome data.

\section{Discussion}

Previous whole-body transcriptome studies of $P$. pseudoannulata identified multiple candidate genes involved in the spider's response to $\mathrm{Cd}$ stress, and also identified that $\mathrm{Cd}$

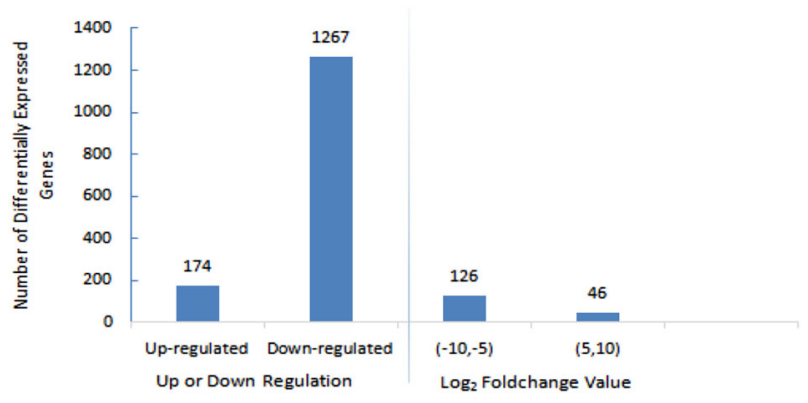

Fig. 3 Distribution of differently expressed unigenes between cerebral ganglia of Cd-treated and control P. pseudoannulata stress causes oxidative damage to digestive tissues and disrupts metabolic activities and signal transduction. Signaling pathways that were affected included Notch, MAPK, AMPK, Hedgehog, Ras and TNF. Cd stress also changed the expression of $\mathrm{ABC}$ transporters, and induced the expression of glutathione metabolism related enzymes, Hsp70 and Hsp20 in the spider (Li et al. 2016). The cerebral ganglion is involved in crucial physiological functions, such as foraging behavior, integrating sensory information, enabling the spider to respond appropriately to environmental stimuli, and ability of the spider to orient itself. In addition, it is well established that the activity of neurosecretory cells in the cerebral ganglion determines molting and sexual maturation (Foelix 2011). To better understand how $\mathrm{Cd}$ affects animals and their interactions with the environment, we sought to characterize the genes affected by $\mathrm{Cd}$ toxicity in the spider's cerebral ganglion at the transcriptome level.

\section{Metabolism}

Oxidative phosphorylation oxidizes nutrients to release ATP, which serve as a source of energy in the cell, and this process primarily occurs in the mitochondria of eukaryotic cells (Rédei 2008). We found that enzymes involved in oxidative phosphorylation processes experienced change in gene expression levels when spiders were treated with $\mathrm{Cd}$. Enzymes included NADH dehydrogenase (ubiquinone), succinate dehydrogenase (ubiquinone) flavoprotein, ubiquinol-cytochrome c reductase, cytochrome c oxidase

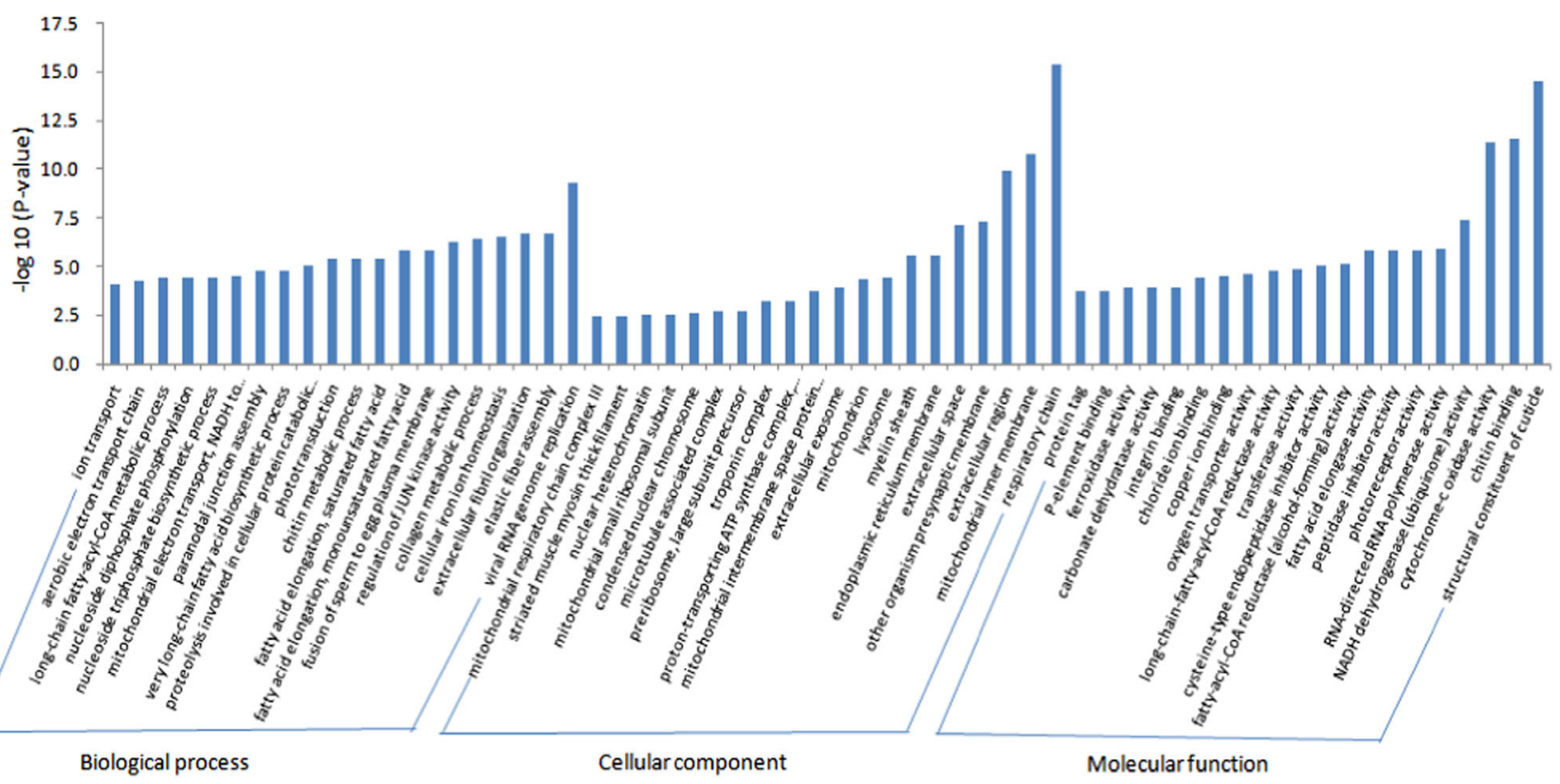

Fig. 4 GO enrichment analysis of top 30 terms represented in the DEGs. The abscissa is the Unigene number, and the vertical axis lists the GO term 
Fig. 5 Enriched KEGG pathways that are represented in the DEGs of Cd-treated and control $P$. pseudoannulata

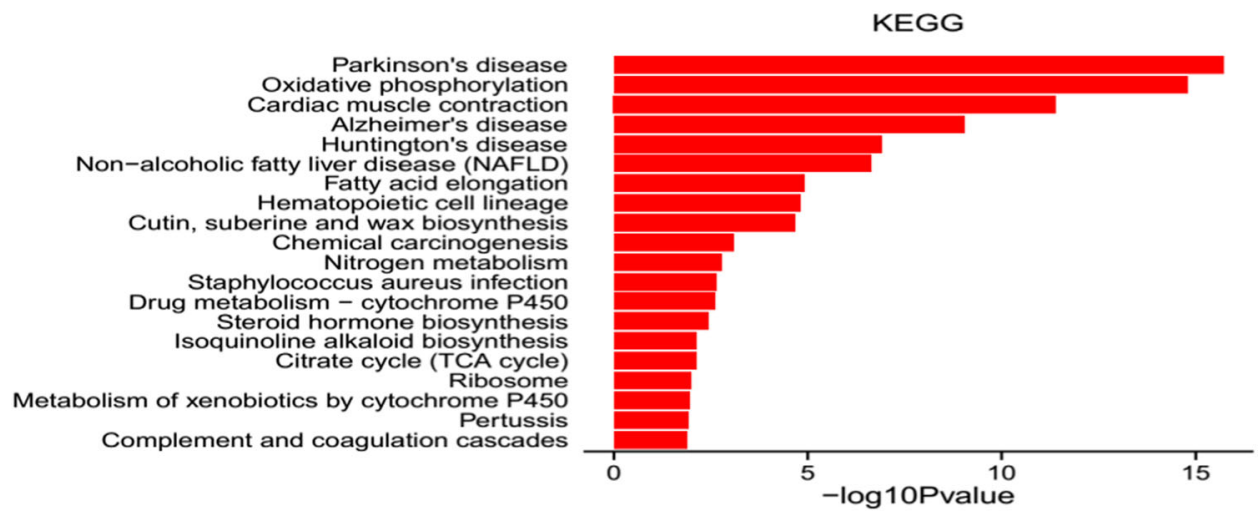

\begin{tabular}{lllll}
\hline Ko ID & Term & Gene number & Enrichment score & Regulated \\
\hline ko04020 & Calcium signaling pathway & 5 & 5.35 & $\mathrm{Up}$ \\
k004022 & cGMP_PKG signaling pathway & 5 & 4.50 & $\mathrm{Up}$ \\
ko00350 & Tyrosine metabolism & 4 & 12.43 & $\mathrm{Up}$ \\
ko04745 & Phototransduction一fly & 4 & 9.51 & $\mathrm{Up}$ \\
k004916 & Melanogenesis & 4 & 6.80 & $\mathrm{Up}$ \\
k000950 & Isoquinoline alkaloid biosynthesis & 4 & 22.19 & $\mathrm{Up}$ \\
ko00190 & Oxidative phosphorylation & 41 & 4.53 & Down \\
k005012 & Parkinson's disease & 41 & 4.19 & Down \\
k005010 & Alzheimer's disease & 34 & 3.00 & Down \\
ko05016 & Huntington's disease & 34 & 2.41 & Down \\
ko04932 & Non-alcoholic fatty liver disease (NAFLD) & 27 & 3.18 & Down \\
k003010 & Ribosome & 24 & 1.66 & Down \\
\hline
\end{tabular}

Table 4 The dominant and significantly enriched KEGG pathways in the DEGs of Cdtreated and control $P$. pseudoannulata cbb3-type and F-type $\mathrm{H}+$-transporting ATPase, which all showed lower expression levels under $\mathrm{Cd}$ stress. Of the OXPHOS-related genes that showed changes in expression after Cd-stress, 9/16 genes encoded NADH dehydrogenase (ubiquinone) and were expressed at lower levels. NADH dehydrogenase (ubiquinone), which is alternatively referred to as Complex I (EC 1.6.5.3) that catalyzes the transfer of electrons from $\mathrm{NADH}$ to coenzyme Q10 (CoQ10) (Nakamaru-Ogiso et al. 2010). Brandt (2006) indicated that Complex I is the largest and most complicated enzyme of the electron transport chain, which is located in the inner mitochondrial membrane. The detoxification processes of $\mathrm{Cd}$ are likely energyintensive and affect the spider's development, reproduction, biochemical and physiological processes (Li et al. 2016; Eraly et al. 2011; Wilczek et al. 2013). Cd may cause damage of nerve cells by reducing the levels of oxidative phosphorylation enzymes in the spider cerebral ganglion, leading to a shortage of energy there. Neurons have an overall high metabolic rate, therefore neurons may suffer more from heavy metal damage (Nava-Ruíz and MéndezArmenta 2013). Sun et al. (2016) found changes in gene expression levels of oxidative phosphorylation enzymes in fresh water crabs when exposed to $\mathrm{Cd}$. Another study also showed that oxidative phosphorylation was critical for spider's to respond to high temperature stress (Xiao et al. 2016). The KEGG annotation of the DEGs identified pathway types for Parkinson's disease (ko05012), Alzheimer's disease (ko05010), and Huntington's disease (ko05016) that were differentially expresed, indicating that $\mathrm{Cd}$ influenced changes in gene expression for genes that are involved in neurological diseases.

The primary effect of $\mathrm{Cd}$ exposure in animals appears to be oxidative stress caused by the accumulation of reactive oxygen species (ROS) (Itziou et al. 2011). Superoxide is a ROS that can lead to oxidative stress in the cell, and its accumulation is also linked to neuromuscular diseases and aging (Esterházy et al. 2008). These results were corroborated by previous studies that showed that $\mathrm{Cd}$ induces oxidative stress and mitochondrial dysfunction, leading to neurodegenerative disorders and nervous function disorders, such as Alzheimer's disease and amyotrophic lateral sclerosis (Jiang et al. 2007; Bar-Sela et al. 2001). NADH dehydrogenase produces superoxide by transferring one electron from FMNH2 to oxygen $\left(\mathrm{O}_{2}\right)$. Since the gene showed strong changes in expression under $\mathrm{Cd}$ stress, 


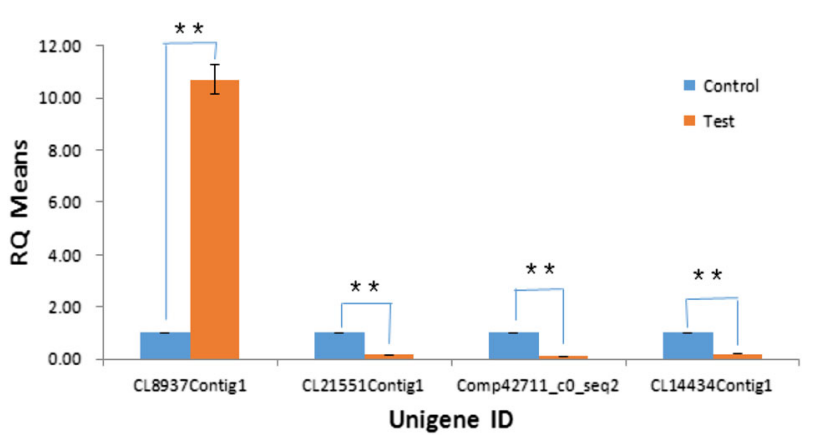

Fig. 6 Validation of the RNA-Seq expression profiles of selected DEGs by qPCR

Cd-induced disruption of the cerebral ganglion of spider may result in disrupting superoxide production.

While most DEGs detected in this study were downregulated, the tyrosine metabolism pathway encoding tyrosinase and tyrosine 3-monooxygenase showed upregulation under $\mathrm{Cd}$ stress. Tyrosinase is involved in wide ranging processes in insects including wound healing and sclerotization, melanin synthesis and parasite encapsulation. Some commonly used insecticides inhibit tyrosinase, implying its importance in insect defensive mechanisms to stress (Kim and Uyama 2005). Nagatsu (1995) suggested that tyrosine 3-monooxygenase was a precursor for neurotransmitters norepinephrine (noradrenaline) and epinephrine (adrenaline), which were produced in the cerebral ganglion, peripheral sympathetic neurons and the adrenal medulla. Given that tyrosine metabolism pathway genes showed increased gene expression under Cd-treated conditions, these genes may play a role in defending spiders against $\mathrm{Cd}$-induced damage to the nervous system.

\section{Signal transduction}

Signal transduction is the process by which a chemical or physical signal is transmitted through a cell as a series of molecular events, and it is the basic mechanism controlling cell growth, proliferation, metabolism and many other processes (Jiang et al. 2007). Signal transduction plays important roles in heavy metal stress, including metal transport, metal regulation, and detoxification functions. (Leonard et al. 2004; Adams et al. 2002; Tian et al. 2015). $\mathrm{Cd}$ can interact with different hormonal signaling pathways, such as estrogen and MAPK signaling that can control proliferation, differentiation, and survival/death (Ali et al. 2010, 2012; Clapham 2007). Cd-stress primarily resulted in changes in gene expression of the calcium signaling pathway and the cGMP-PKG signaling pathway (Table 4). As a ubiquitous second messenger, calcium $\left(\mathrm{Ca}^{2+}\right)$ plays a significant role in cellular signaling, and is particularly involved in neuronal functions such as muscle contractions, neuronal transmission, neurogenesis, and synaptic plasticity (Demaurex and Nunes 2016; Rash et al. 2016; Berridge et al. 2000). $\mathrm{Ca}^{2+}$ signaling is involved in response to metal stress in both plants and animals (Wang et al. 2011; Wang et al. 2014). Sun (2011) suggested that $\mathrm{Ca}^{2+}$ plays a crucial role in the apoptosis of rat cortical neurons induced by $\mathrm{Cd}$ exposure. KEGG analysis of DEGs identified the upregulation of phosphatidylinositol phospholipase (PLCB), $\mathrm{Ca}^{2+}$ transporting ATPase (ATP2A) and solute carrier family 25 (ANT), and GO annotation of the enriched DEGs identified 14 GO terms including 20 genes related to "calcium signal". These results suggest that the calcium signaling pathway could be actived or become regulated downstream of another pathway to contribute to the response of spider cerebral ganglia under metal stress. This result was consistent with the calcium signaling pathway quickly and effectively regulating downstream signaling and gene expression in response to low temperature stress in P. pseudoannulata (Xiao et al. 2016).

As noted above, one of the pathways that were significantly up-regulated under $\mathrm{Cd}$ stress was the the cGMPPKG signaling pathway. Specifically, phosphoinositide phospholipase C (PLC) (EC 3.1.4.11) and $\mathrm{Ca}^{2+}$ transporting ATPase (PMCA) (EC:3.6.3.8) were significantly upregulated in the cerebral ganglion of spiders when exposed to Cd. PLC belongs to a larger superfamily of phospholipases, which is a family of eukaryotic intracellular enzymes that plays an important role in signal transduction processes (Meldrum et al. 1991). PLC is an enzyme that facilitates the release of $\mathrm{Ca}^{2+}$, leading to increased intracellular $\mathrm{Ca}^{2+}$ levels, and facilitating cellular responses through stimulation of $\mathrm{Ca}^{2+}$-sensitive proteins such as Calmodulin. PMCA is a $\mathrm{Ca}^{2+}$ transport ATPase that localizes to the plasma membrane and facilitates the removal of $\mathrm{Ca}^{2+}$ from the cell, playing a viral role in regulating $\mathrm{Ca}^{2+}$ levels within cells (Jensen et al. 2004). PMCA is placed within three KEGG pathways that were significantly enriched, including calcium signaling pathway, cGMP-PKG signaling pathway, both of which were up-regulated, and Alzheimer's disease, which was down-regulated. These results suggest that PMCA may be a key protein involved in response to $\mathrm{Cd}$ exposure in the cerebral ganglion of spiders. However, the underlying mechanism of metalloregulatory and detoxification in cerebral ganglion of spider under $\mathrm{Cd}$ stress at the protein level needs to be further studied.

In our analysis, we found up-regulation of genes encoding phototransduction-fly signaling related proteins, suggesting that $\mathrm{Cd}$ may interfere with the optical signal transduction in the spider. This is consistent with results of previous studies in which spiders exposed to $\mathrm{Cd}$ exhibited increased apoptosis in photoreceptors and ganglionic cells that led to phenotypic changes (Roozbehi et al. 2007). 


\section{Genetic information processing}

From the KEGG analysis of DEGs, 24 ribosome proteins were down-regulated in the cerebral ganglion of spiders exposed to $\mathrm{Cd}$. The spider cerebral gaglion releases acetylcholine, approximately 10 different amino acids and several biogenic amines as neurotransmitters (Schmid et al. 1992). The ribosomal proteins levels decrease in spider exposed to environmental stress, such as high temperatures (Xiao et al. 2016), indicating which contributed to protein biosynthesis compensates upon stress. The down-regulation of ribosomal proteins may lead to damage in the spider cerebral ganglion by disrupting the synthesis of proteins related to neurotransmitter regulation.

\section{Conclusion}

In summary, we generated transcriptomes of the cerebral ganglion of $P$. pseudoannulata under standard lab conditions, and under Cd stress. A total of 123,328 assembled unigenes were obtained, with an average length of 1040.73 bp. We identified $1441 \mathrm{Cd}$ stress-associated DEGs in the cerebral ganglion of $P$. pseudoannulata, and 39 different significantly enriched pathways involving metabolic processes, signaling transduction, protein processing, and nervous diseases. We found that exposure to $\mathrm{Cd}$ leads to changes in the expression of genes involved in oxidative phosphorylation-associated, calcium signaling-associated, cGMP-PKG signaling, and nervous disease-associated and ribosomal-associated genes. Overall, the our results facilitated the discovery of genes that respond to the neurotoxic effects of $\mathrm{Cd}$ and in the identification of biomarkers for monitoring heavy metal pollution.

\footnotetext{
Acknowledgements This work was supported by the National Natural Science Foundation of China (No. 31472017; 81371836; 81572023), Project of Science and Technology Department of Hunan Province (No. 2014FJ2003), Scientific research project of Hunan provincial education department (16C0778), Grants from the National Key Research and Development Program of China (2016YFC1202003), Guangdong Natural Science Foundation (2014A030313134), Science and Technology Planning Project of Guangdong Province (2016A050502008), the Project of Basic Platform of National Science and Technology Resources of the Ministry of Sciences and Technology of China (TDRC-2017-22) and 111 Project (B12003), the Agricultural Science and Technology Innovation Program of China (No. CAAS-ASTIP-2017-IBFC), and 2016 Youth Fund Project of College of Orient Science \& Technology of Hunan Agriculture University (16QNZ07). Mention of trade names or commercialproducts in this article is solely for the purpose of providing specific information and does not imply recommendation or endorsement by the U.S. Department of Agriculture. All programs and services of the U.S. Department of Agriculture are offered on a nondiscriminatory basis without regard to race, color, national origin, religion, sex, age, marital status, or handicap.
}

Author contributions Z.W. and Z.Y.L. conceived and designed the study. H.L.Y. and Y.D.P. performed the research. Y.X.S., X.J.P., C.L. X., X.X. and J.X.T. collected data, performed modeling work. Y.D.P., J.L.H and J.W. analyzed output data. Q.S.S. revised the manuscript. H. L.Y. wrote the manuscript, and all authors contributed substantially to revisions. All the authors read and approved the final manuscript.

\section{Compliance with ethical standards}

Conflict of interest The authors declare that they have no conflict of interest.

Ethical approval Materials used in this study were unregulated common arthropod spider and Drosophila melanogaster.

Open Access This article is distributed under the terms of the Creative Commons Attribution 4.0 International License (http://crea tivecommons.org/licenses/by/4.0/), which permits unrestricted use, distribution, and reproduction in any medium, provided you give appropriate credit to the original author(s) and the source, provide a link to the Creative Commons license, and indicate if changes were made.

\section{References}

Adams TK, Saydam N, Steiner F, Schaffner W, Freedman JH (2002) Activation of gene expression by metal-responsive signal transduction pathways. Environ Health Persp 5:813-817

Ali I, Damdimopoulou P, Stenius U, Adamsson A, Mäkelä SI, Åkesson A, Berglund M, Håkansson H, Halldin K (2012) Cadmium-induced effects on cellular signaling pathways in the liver of transgenic estrogen reporter mice. Toxicol Sci 127 (1):66-75

Ali I, Penttinen-Damdimopoulou PE, Mäkelä SI, Mäkelä SI, Berglund M, Stenius U, Åkesson A, Håkansson H, Halldin K (2010) Estrogen-like effects of cadmium in vivo do not appear to be mediated via the classical estrogen receptor transcriptional pathway. Environ Health Persp 118(10):1389-1394

Bar-Sela S, Reingold S, Richter ED (2001) Amyotrophic lateral sclerosis in a battery-factory worker exposed to cadmium. Int $\mathrm{J}$ Occup Environ Health 7:109-112

Berridge MJ, Lipp P, Bootman MD (2000) The versatility and universality of calcium signalling. Nat Rev Mol Cell Bio 1(1):11-21

Brandt U (2006) Energy converting NADH:quinone oxidoreductase (complex I). Annu Rev Biochem 75:69-92

Clapham DE (2007) Calcium signaling. Cell 131:1047-1058

Conesa A, Götz S, García-Gómez JM, Terol J, Talón M, Robles M (2005) Blast2GO: a universal tool for annotation, visualization and analysis in functional genomics research. Bioinformatics 21 (18):3674-3676

Demaurex N, Nunes P (2016) The role of STIM and ORAI proteins in phagocytic immune cells. Am J Physiol Cell Physiol 310(7): C496-C508

Eraly D, Hendrickx F, Backeljau T, Bervoets L, Lens L (2011) Direct and indirect effects of metal stress on physiology and life history variation in field populations of a lycosid spider. Ecotoxicol Environ Saf 74:1489-1497

Eraly D, Hendrickx F, Bervoets L (2010) Experimental exposure to cadmium affects metallothionein-like protein levels but not survival and growth in wolf spiders from polluted and reference populations. Environ Pollut 158(6):2124-2131 
Esterházy D, King MS, Yakovlev G, Hirst J (2008) Production of reactive oxygen species by complex I (NADH:ubiquinone oxidoreductase) from Escherichia coli and comparison to the enzyme from mitochondria. Biochemistry 25(12):3964-3971

Foelix RF (2011) Biology of spiders. Oxford University Press, Oxford Gerhard K (2008) Biochemistry of signal transduction and regulation. Wiley-VCH, Weinheim, Germany, p 15

Grabherr MG, Haas BJ, Yassour M et al. (2011) Trinity: reconstructing a full-length transcriptome without a genome from RNA-Seq data. Nat Biotechnol 29(7):644-652

Habeebu SS, Liu Y, Park JD, Klaassen CD (2001) Strain differences in the toxicity of cadmium to trigeminal ganglia in mice. Toxicol Appl Pharmacol 177(3):200-207

Itziou A, Kaloyianni M, Dimitriadis VK (2011) In vivo and in vitro effects of metals in reactive oxygen species production, protein carbonylation, and DNA damage in land snails Eobania vermiculata. Arch Environ Contam Toxicol 60:697-707

Jensen TP, Buckby LE, Empson RM (2004) Expression of plasma membrane $\mathrm{Ca}^{2+}$ ATPase family members and associated synaptic proteins in acute and cultured organotypic hippocampal slices from rat. Brain Res Dev Brain Res 152(2):129-136

Jiang LF, Yao TM, Zhu ZL, Wang C, Ji LN (2007) Impacts of Cd(II) on the conformation and self-aggregation of Alzheimer's tau fragment corresponding to the third repeat of microtubule-binding domain. Biochim Biophys Acta 1774:1414-1421

Jöst C, Zauke GP (2008) Trace metal concentrations in Antarctic sea spiders (Pycnogonida, Pantopoda). Mar Pollut Bull 56 (8):1396-1399

Jung CS, Lee SB, Jung MP, Lee SH (2005) Accumulated heavy metal content in wolf spider, Pardosa astrigera (Araneae: Lycosidae), as a bioindicator of exposure. J Asia Pac Entomol 8:185-192

Jung MP, Lee JH (2012) Bioaccumulation of heavy metals in the wolf spider, Pardosa astrigera L. Koch (Araneae: Lycosidae). Environ Monit Assess 184(3):1773-1779

Kim YJ, Uyama H (2005) Tyrosinase inhibitors from natural and synthetic sources: structure, inhibition mechanism and perspective for the future. Cell Mol Life Sci 62(15):1707-1723

Leonard SS, Harris GK, Shi X (2004) Metal-induced oxidative stress and signal transduction. Free Radic Biol Med 37(12):1921-1942

Li CC, Li GY, Yun YL, Chen J, Zhang ZT, Peng Y (2016) The effect of cadmium exposure on fitness-related traits and antioxidant responses in the wolf spider, Pardosa pseudoannulata. Bull Environ Contam Toxicol 97:31-36

Li CC, Wang Y, Li GY, Yun YL, Dai YJ, Chen J, Peng Y (2016) Transcriptome profiling analysis of wolf spider Pardosa pseudoannulata (Araneae: Lycosidae) after cadmium exposure. Int $\mathrm{J}$ Mol Sci 17(12):2033

Li JQ, Zhao ZM, Zhou JJ (2001) Advances in the studies of spiders in rice field. Acta Arachnoiogica Sin 10(2):58-63. (in Chinese with English summary)

Liu HL, Zheng LM, Liu QQ, Quan FS, Zhang Y (2013) Studies on the transcriptomes of non-model organisms. Hereditas 35 (8):955-970

Margulies M, Egholm M, Altman WE et al. (2005) Genome sequencing in microfabricated high-density picolitre reactors. Nature 437(7057):376-380

Mehinto AC, Pruchab MS, Colli-Dulac RC, Kroll KJ, Lavelle CM, Barberc DS, Vulpe CD, Denslowc ND (2014) Gene networks and toxicity pathways induced by acute cadmium exposure in adult largemouth bass (Micropterus salmoides). Aquat Toxicol 152:186-194

Meldrum E, Parker PJ, Carozzi A (1991) The PtdIns-PLC superfamily and signal transduction. Biochim Biophys Acta 1092(1):49-71

Meng XL, Liu M, Jiang KY, Wang BJ, Tian X, Sun SJ, Luo ZY, Qiu CW, Wang L (2013) De novo characterization of Japanese scallop Mizuhopecten yessoensis transcriptome and analysis of its gene expression following cadmium exposure. PLoS ONE 8(5): e64485

Murthy RC, Saxena DK, Lal B, Chandra SV (1989) Chronic cadmium-ethanol administration alters metal distribution and some biochemicals in rat brain. Biochem Int 19(1):135-143

Nagatsu T (1995) Tyrosine hydroxylase: human isoforms, structure and regulation in physiology and pathology. Essays Biochem 30:15-35

Nakamaru-Ogiso E, Han H, Matsuno-Yagi A, Keinan E, Sinha SC, Yagi T, Ohnishi T (2010) The ND2 subunit is labeled by a photoaffinity analogue of asimicin, a potent complex I inhibitor. FEBS Lett 584(5):883-888

Nava-Ruíz C, Méndez-Armenta M (2013) Cadmium, lead, thallium: occurrence, neurotoxicity and histopathological changes of the nervous system. Pollut Dis Remediat Recycl 4:321-349

Nemmiche S, Chabane-Sari D, Kadri M, Guiraud P (2011) Cadmium chloride-induced oxidative stress and DNA damage in the human Jurkat $\mathrm{T}$ cell line is not linked to intracellular trace elements depletion. Toxicol Vitr 25:191-198

Pertea G, Huang X, Liang F et al. (2003) TIGR gene indices clustering tools (TGICL): a software system for fast clustering of large EST datasets. Bioinformatics 19(5):651-652

Rash BG, Ackman JB, Rakic P (2016) Bidirectional radial $\mathrm{Ca}^{2+}$ activity regulates neurogenesis and migration during early cortical column formation. Sci Adv 2(2):e1501733

Roozbehi A, Almasi-Tork S, Piryaee A, Sadeghi Y (2007) Effects of cadmium on photoreceptors and ganglionic cells of retinal layer in mice embryo-an ultrastructural study. Indian J Exp Biol 45 (5):469-474

Rédei GP (2008) Oxidative phosphorylation (OXPHOS). Springer, The Netherlands, pp 1418-1418

Samu F, Szinetár C (2002) On the nature of agiobiont spiders. J Arachnol 30(2):389-402

Schmid A, Sperk G, Reither H (1992) Reither: quantitative determination of neuroactive substances in the CNS of the spider Cupiennius salei keys. Comp Biochem Physiol 102:447-450

Sun M, Li YT, Liu Y, Chin LS, Wang L (2016) Transcriptome assembly and expression profiling of molecular responses to cadmium toxicity in hepatopancreas of the freshwater crab Sinopotamon henanense. Sci Rep 6:19405

Sun Y (2011) Effects of calcium on cadmium induced in vitro culture of rat cerebral cortex. The role of neuronal apoptosisr. Yangzhou University, Yangzhou, China

Tatusov RL, Fedorova ND, Jackson JD et al. (2003) The COG database: an updated version includes eukaryotes. BMC Bioinforma 4 (1):41

Tian SQ, Gu C, Liu LQ, Zhu XD, Zhao YH, Huang SZ (2015) Transcriptome profiling of Louisiana iris root and identification of genes involved in lead-stress response. Int $\mathrm{J}$ Mol Sci 16:28087-28097

Trapnell C, Williams BA, Pertea G, Mortazavi A, Kwan G, van Baren MJ, Salzberg SL, Wold BJ, Pachter L (2010) Transcript assembly and quantification by RNA-Seq reveals unannotated transcripts and isoform switching during cell differentiation. Nat Biotechnol 28(5):511-515

Wang J, Peng YD, He C, Wei BY, Liang YS, Yang HL, Wang Z, Stanley D, Song QS (2016a) Cry1Ab-expressing rice did not influence expression of fecundity-related genes in the wolf spider Pardosa pseudoannulata. Gene 592:1-7

Wang J, Peng YD, Xiao KF, Wei BY, Jilin Hu, Wang Z, Song QS, Zhou XG (2017) Transcriptomic response of wolf spider, Pardosa pseudoannulata, to transgenic rice expressing Bacillus thuringiensis $\mathrm{Cry} 1 \mathrm{Ab}$ protein. BMC Biotechnol 17:7

Wang JC, Sun Y, Song RL, Bian JC, Liu ZP (2011) Cadmium induced apoptosis in rat hepatocytes by calcium overloading. Acta Vet Zootech Sin 42(8):1168-1174 
Wang L, Feng Z, Wang X, Zhang X (2010) DEGseq: an R package for identifying differentially expressed genes from RNA-seq data. Bioinformatics 26(1):136-138

Wang XH, Liu H, Yi HL (2014) Involvement of ROS and calcium in cadmium-induced yeast cell death. Acta Sci Circumstantiae 34 (7):1869-1873

Wang ZH, Shao Y, Li CH, CH J (2016b) RNA-seq analysis revealed ROS-mediated related genes involved in cadmium detoxification in the razor clam Sinonovacula constricta. Fish Shellfish Immunol 57:350-361

Wicker T, Schlagenhauf E, Graner A et al. (2006) 454 sequencing put to the test using the complex genome of barley. BMC Genom 7 (1):275

Wilczek G, Babczyn’ska A, Wilczekb P, Doleżych B, Migula P, Młyńska H (2008) Cellular stress reactions assessed by gender and species in spiders from areas variously polluted with heavy metals. Ecotoxical Environ Saf 70(1):127-137

Wilczek G, Babczýnska A, Wilczek P (2013) Antioxidative responses in females and males of the spider Xerolycosa nemoralis (Lycosidae) exposed to natural and anthropogenic stressors. Comp Biochem Physiol C 157:119-131

Xiao R, Wang L, Cao YH, Zhang G (2016) Transcriptome response to temperature stress in the wolf spider Pardosa pseudoannulata (Araneae: Lycosidae). Ecol Evol 6(11):3540-3554

Yang HL, Peng YD, Tian JX, Wang J, Hu JL, Wang Z (2016) Spiders as excellent experimental models for investigation of heavy metal impacts on the environment: a review. Environ Earth Sci 75:1059

Ye J, Fang L, Zheng HK et al (2006) WEGO: a web tool for plotting GO annotations. Nucleic Acids Res 34(Web Server issue): W293-W297 